\title{
Periodic Correlation Structures in Bacterial and Archaeal Complete Genomes
}

\author{
Zuo-Bing $\mathrm{Wu}^{*}$
}

State Key Laboratory of Nonlinear Mechanics, Institute of Mechanics, Chinese Academy of Sciences, Beijing 100190, China

\begin{abstract}
The periodic transference of nucleotide strings in bacterial and archaeal complete genomes is investigated by using the metric representation and the recurrence plot method. The generated periodic correlation structures exhibit four kinds of fundamental transferring characteristics: a single increasing period, several increasing periods, an increasing quasi-period and almost noincreasing period. The mechanism of the periodic transference is further analyzed by determining all long periodic nucleotide strings in the bacterial and archaeal complete genomes and is explained as follows: both the repetition of basic periodic nucleotide strings and the transference of non-periodic nucleotide strings would form the periodic correlation structures with approximately the same increasing periods.
\end{abstract}

Keywords: Bacterial and archaeal complete genomes, periodic correlation structures, metric representation, recurrence plots.

\section{INTRODUCTION}

Since complete genomes of many organisms are available from web-based databases, a full and systematic search of genome structures, functions and dynamics becomes an essential part of the study for both biologists and physicists. For the large amount of genomes, developing quantitative methods to extract meaningful information is a major challenge with respect to applications of statistical mechanics and nonlinear dynamics to biological systems [1, $2]$. To understand the complete genomes, some statistical and geometrical methods were developed [3-18]. The studies of the complete genomes of many organisms came up with the determinations of the nontrivial statistical characteristics, such as the long-range correlations, the short-range correlations and the fractal features or genomic signatures. In particular, it was found that the transposable elements, as the mobile DNA sequences, have the ability to move from one place to another and make many replicas within the genome via the transposition [19-21]. Their origin, evolution, and tremendous effects on the genome structure and the gene function are issues of fundamental importance in biology [22-24].

In general, the symbolic dynamics and the recurrence plots are basic methods of nonlinear dynamics for analyzing complex systems [25, 26]. Although the conventional methods have made great strides in understanding genetic patterns, they are requried to analyze the so-called junk DNA with complex functions governing mutations [27, 28]. Recently, a one-to-one metric representation of a genome borrowed from the symbolic dynamics was proposed to form a fractal pattern in a plane $[29,30]$. By using the metric representation method, the recurrence plot technique of the

*Address correspondence to this author at the State Key Laboratory of Nonlinear Mechanics, Institute of Mechanics, Chinese Academy of Sciences, Beijing 100190, China; Tel: 86-10-82543955; Fax: 86-1082543977; E-mail: wuzb@Inm.imech.ac.cn genome was established to analyze the correlation structures of nucleotide strings [31,32]. The transference of nucleotide strings appears at many positions of a complete genome and makes a regular and irregular correlation structures, but the periodic correlation structures in the complete genome are the most interesting in view of the dynamics. In this paper, using the metric representation and the recurrence plot method, we identify periodic correlation structures in bacterial and archaeal complete genomes and analyze the mechanism of the periodic correlation structures. Since the nucleotide strings include transposable elements, the mechanism is conducible to understanding the genome structures in terms of nucleotide strings transferring in the genomes and exploring relations between transference of nucleotide strings and the transposable elements.

\section{CORRELATION STRUCTURES IN PERIODIC AND RANDOM SEQUENCES}

In what follows, we give a brief presentation of the metric representation and the recurrence plot method, which are detailed in [29-32]. For a given symbolic sequence $s_{1} s_{2} \ldots s_{i} \ldots s_{N}\left(s_{i} \in\{A, C, G, T\}\right)$, a metric representation for its subsequences $\sum_{k}=s_{1} s_{2} \ldots s_{k}(1 \leq k \leq N)$ is defined as

$\alpha_{k}=2 \sum_{j=1}{ }^{k} \mu_{k-j+1} 3^{-j}+3^{-k}=2 \sum_{i=1}{ }^{k} \mu_{i} 3^{-(k-i+1)}+3^{-k}$,

$\beta_{k}=2 \sum_{j=1}^{k} v_{k-j+1} 3^{-j}+3^{-k}=2{ }_{i=1}^{k} v_{i} 3^{-(k-i+1)}+3^{-k}$,

where $\mu_{i}$ is 0 if $s_{i} \in\{A, C\}$ or 1 if $s_{i} \in\{G, T\}$ and $v_{i}$ is 0 if $s_{i} \in\{A, T\}$ or 1 if $s_{i} \in\{C, G\}$. It maps the one-dimensional symbolic sequence to the two-dimensional plane $(\alpha, \beta)$. The subsequences with the same ending $l$-nucleotide string are labeled with $\sum^{l}$. They correspond to points in the zone encoded by the $l$-nucleotide string. With two subsequences $\sum_{i} \in \sum^{l}$ and $\sum_{j}(j \geq l)$, we calculate

$\Theta\left(\varepsilon_{l}-\left|\sum_{i}-\sum_{j}\right|\right)=\Theta\left(\varepsilon_{l}-\left[\left(\alpha_{i}-\alpha_{j}\right)^{2}+\left(\beta_{i}-\beta_{j}\right)^{2}\right]^{1 / 2}\right)$,

where $\varepsilon_{l}=1 / 3^{l}$ and $\Theta$ is the Heaviside function $[\Theta(x)=1$, if $x$ $>0 ; \Theta(x)=0$, if $x \leq 0]$. When $\Theta\left(\varepsilon_{l}-\left|\sum_{i}-\sum_{j}\right|\right)=1$, i.e., $\sum_{j} \in \sum^{l}$, a point $(i, j)$ is plotted on a plane. Repeating the above process 
for $i \in[l, N]$ and $j \in[l, N]$, we obtain a recurrence plot of the symbolic sequence. To present the correlation structure in the recurrence plot plane, we define a correlation intensity at a given correlation distance $d$ as

$\Xi(d)=\sum_{i=1}^{N-d} \Theta\left(\varepsilon_{l}-\left|\sum_{i}-\sum_{i+d}\right|\right)$,

which displays the transference of $l$-nucleotide strings in the symbolic sequence. On the recurrent plot plane, since $\sum_{i}$ and $\sum_{j} \in \sum^{l}$, the transferring element has a length $l$ at least. We calculate the maximal value of $x$ to satisfy

$\Theta\left(\varepsilon_{l}-\left|\sum_{i+x}-\sum_{j+x}\right|\right)=1, x=0,1,2, \ldots x_{\max }$,

i.e., $\sum_{i+x}$ and $\sum_{j+x} \in \sum^{l}$. The transferring element has a length $L=l+x_{\max }$ and is placed at the positions $\left(i-l+1, i+x_{\max }\right)$ and $(j-$ $\left.l+1, j+x_{\text {max }}\right)$, which implies the correction distance $d=j-i$.

To understand the transferring characteristics of a complex genome, we investigate the correlation structures of simple periodic and random sequences. By randomly combining the four letters $A, C, G$ and $T$, we firstly generate two random nucleotide sequences: one has the length of 67 and another has the length of 5000. Then, a periodic nucleotide sequence with the total length of 5000 is formed by repeating the short nucleotide string. Using the metric representation and the recurrence plot method, we may determine the correlation intensities at different correlation distances with $l=8$ for the periodic and random sequences in Fig. (1). It is evident that there exist equidistant parallel lines with a basic correlation distance in Fig. (1a), to form the periodic correlation structure for the periodic sequence. The basic correlation distance hereinafter called the basic periodic length is determined as $d_{b}=67$. The correlation intensity $\Xi(d)$ decreases linearly with the increase of the correlation distance $\left(d_{b}, 2 d_{b}, \ldots\right)$. However, in Fig. (1b), the correlation intensity $\Xi(d)$ is very small, so there are almost no correlation structures for the random sequence. Therefore, the periodic and random sequences exhibit two very different transferring characteristics: with the periodic correlation structure (with a linearly decreasing intensity) and without a clear correlation structure.

\section{PERIODIC NUCLEOTIDE STRINGS IN BACTE- RIAL AND ARCHAEAL COMPLETE GENOMES}

At the end of 1999, complete genomes including more of 20 bacteria were in the Genbank [7]. By using the string composition and the metric representation method, the suppressions of all short strings in 23 bacterial and archaeal complete genomes were determined [7,30]. In this section, using the metric representation and the recurrence plot method, we determine all long periodic nucleotide strings $(\geq$ 20 bases) in the 23 bacterial and archaeal genomes. For the 23 genomes, only 13 have long periodic nucleotide strings. All basic strings and their lengths of the long periodic nucleotide strings in the 13 bacterial and archaeal genomes are presented in Table $\mathbf{1}$ in the order of decreasing suppressions of nucleotide strings [30]. Several periods and different basic strings can be seen depending on the genomes, but not necessarily on the lengths of genomes. The genomes of Helicobacter pylori $26695(h p y l)$, Helicobacter pylori J99 (hpyl99), Haemophilus influenzae Rd KW20 (hinf), Mycobacterium tuberculosis H37Rv (mtub),
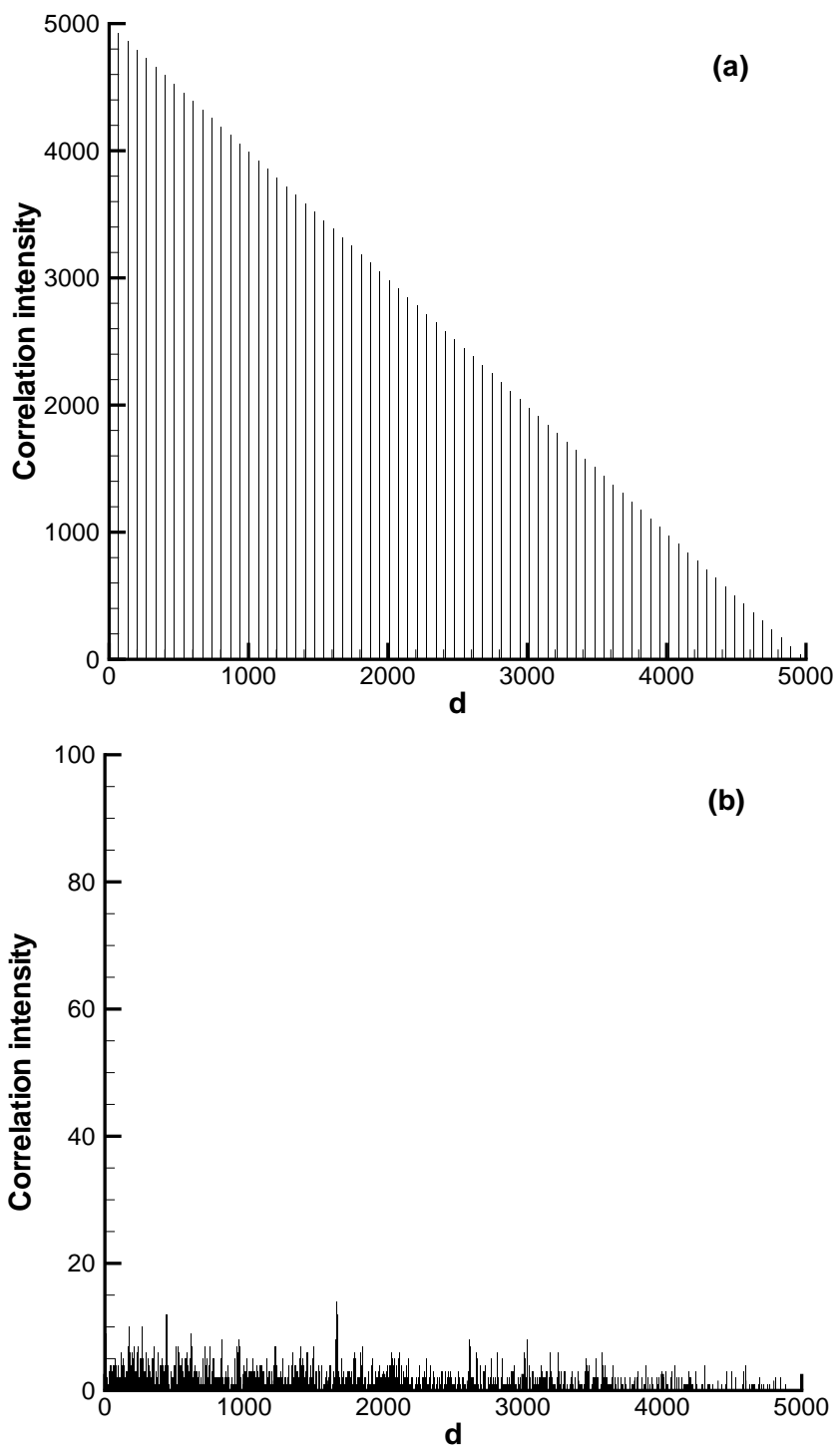

Fig. (1). Plots of correlation intensity $\Xi(d)$ versus correlation distance $d$ for (a) periodic and (b) random sequences.

Synechocystis sp. PCC6803 (synecho) have more periods ( $\geq$ 6 ) and basic strings $(\geq 9)$ than others, which have only fewer periods $(\leq 3)$ and basic strings $(\leq 4)$. In each period, the number of the basic strings generally depends on the length of the period. The longer/shorter period the basic strings have, the smaller/greater their number will be. In the next section, we will investigate the periodic transference of nucleotide strings in the bacterial and archaeal complete genomes and analyze the effects of periodic nucleotide strings on the correlation structures.

\section{PERIODIC CORRELATION STRUCTURES IN BACTERIAL AND ARCHAEAL COMPLETE GENOMES}

The periodic correlation structures of a complete genome contain several basic periodic and/or quasi-periodic lengths, which are determined by using the metric representation and the recurrence plot method as follows. From the relationship between the correlation intensity and the correlation distance 
Table 1. Basic Strings and their Lengths of Long Periodic Nucleotide Strings ( $\geq 20$ bases) in Bacterial and Archaeal Complete Genomes. $N_{p}$ is the Number of the Periods. $N_{s}$ is the Total Number of the Basic Strings

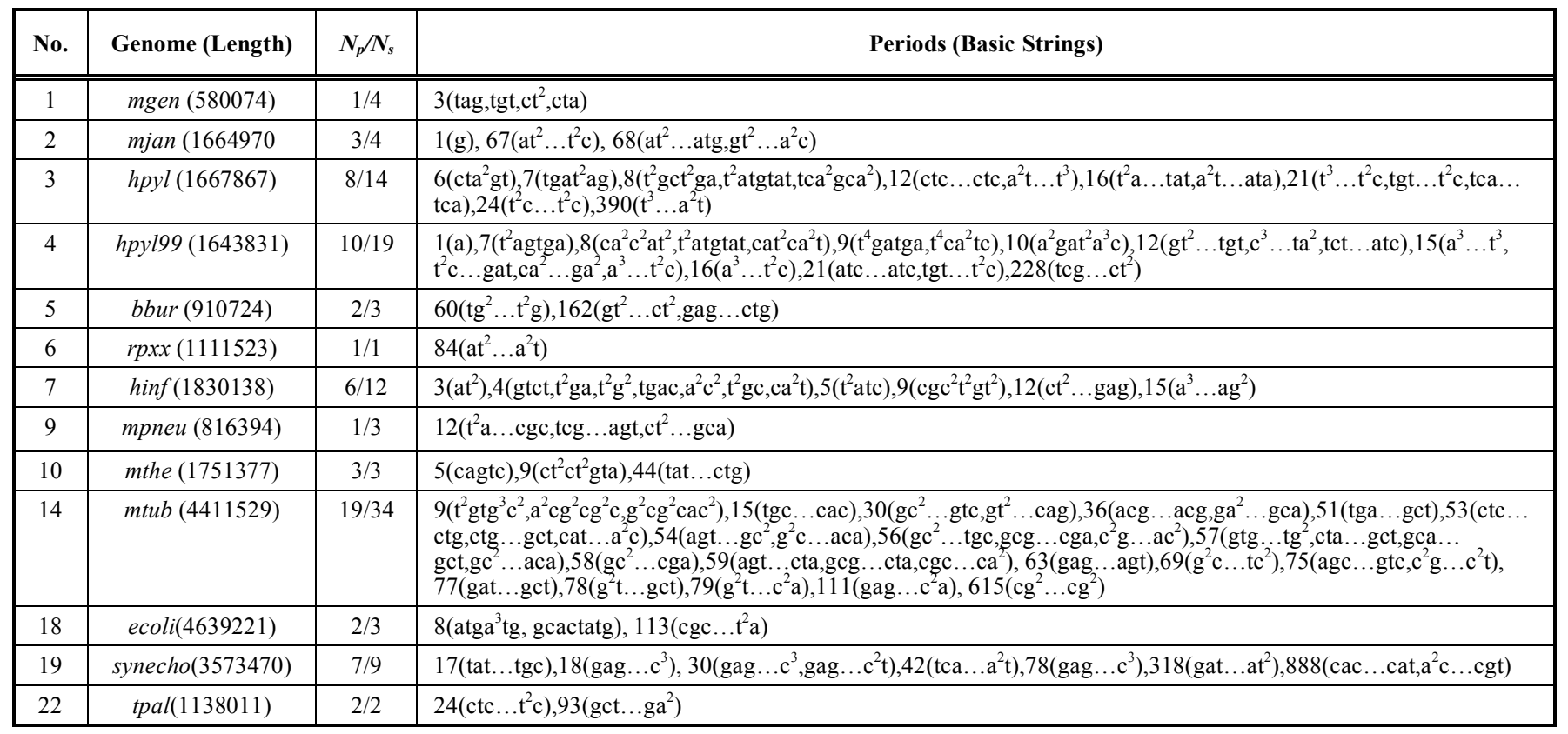

obtained by using Eq. (3), the basic periodic lengths and their integer multiples with strong correlation intensities can be calculated. Moreover, in the transference of nucleotide strings obtained by using Eq. (4), the correlation distance with basic periodic lengths and their integer multiples can also be found. By using both methods, the basic periodic lengths of the periodic correlation structures are determined, as shown in Table 2 , where the 23 complete genomes with

Table 2. Basic Periodic Lengths of Periodic Correlation Structures and Basic String Lengths of Long Periodic Nucleotide Strings ( $\geq 20$ Bases) in Bacterial and Archaeal Complete Genomes Denoted by $d_{b}$ and $p$, Respectively

\begin{tabular}{|c|c|c|c|}
\hline No. & Genome (Acc. No.) & $d_{b}$ & $p$ \\
\hline 1 & mgen (L43967) & 3 & 3 \\
\hline 2 & mjan (L77117) & 67 & $1,67,68$ \\
\hline 3 & hpyl (AE000511) & $7,8, \approx 114$ & $6,7,8,12,16,21,24,390$ \\
\hline 4 & hpyl99 (AE001439) & $8,15,21$ & $1,7,8,9,10,12,15,16,21,228$ \\
\hline 5 & bbur (AE000783) & 162 & 60,162 \\
\hline 7 & $\operatorname{hinf}(\mathrm{L} 42023)$ & 4 & $3,4,5,9,12,15$ \\
\hline 8 & $p N G R 234$ (U00090) & $(6)$ & - \\
\hline 9 & трпеи (U00089) & 12 & 12 \\
\hline 10 & mthe (AE000666) & 67 & $5,9,44$ \\
\hline 11 & aquae (AE000657) & $(67)$ & - \\
\hline 15 & pabyssi (AL096836) & 67 & - \\
\hline 16 & tmar (AE000512) & 67 & - \\
\hline 17 & српеи (AE001363) & $(330)$ & - \\
\hline 18 & ecoli (U00096) & 100,113 & 8,113 \\
\hline 19 & synecho $(\mathrm{BA} 000022)$ & $6,888, \approx 296$ & $17,18,30,42,78,318,888$ \\
\hline 20 & ctra (AE001273) & $(108),(150)$ & - \\
\hline 21 & aero (BA000002) & 65 & - \\
\hline 22 & tpal (AE000520) & 24 & 24,93 \\
\hline 23 & bsub (AL009126) & $\approx 5000$ & - \\
\hline
\end{tabular}


official Genbank accession numbers are arranged in the order of decreasing suppressions of nucleotide strings [30]. When the periodic correlation structures have only a few peaks of the correlation intensity within the correlation distance, the basic periodic lengths are put in parentheses. To see the characteristics of the periodic correlation structures, we also present all basic string lengths in long periodic nucleotide strings ( $\geq 20$ bases) in Table 2 . When a periodic correlation structure is identified based on a long periodic nucleotide string, the transference of nucleotide strings composed of the basic strings appears at some positions where the correlation distance is integer multiples of the period and monotonically increases. At the same time, the lengths of transferred nucleotide strings monotonically decrease. There exists a "cascade" arrangement of nucleotide strings related to the basic periodic length. However, when a periodic correlation structure is identified based on nonperiodic nucleotide strings, the transference of nucleotide strings appears at several positions where the correlation distance is almost integer multiples of the basic periodic length. There are no "cascade" arrangements of nucleotide strings related to the basic periodic length. According to the characteristics of the periodic correlation structures, the results can be summarized as follows:

(1) The correlation distance contains a single increasing period. The most of the complete genomes with a single increasing period have a basic periodic length of 67. They include Methanococcus jannaschii DSM 2661 (mjan), Methanobacterium thermoautotrophicum str. delta $\mathrm{H}$ (mthe), Pyrococcus horikoshii OT3 (pyro), Archaeoglobus fulgidus DSM 4304 (aful), Pyrococcus abyssi (pabyssi) and Thermotoga maritima MSB8 (tmar) genomes. Consider the mjan genome as an example. Fig. (2) displays the correlation intensity at different correlation distances with $l=15$ for the mjan genome. It is evident that there exist some equidistant parallel lines with a basic periodic length, to form a periodic correlation structure. The basic periodic length is determined as $d_{b}=67$. Generally, if the genome has a periodic nucleotide string with the basic string length $p=d_{b}$, it would tend to form a periodic correlation structure. In Table $\mathbf{2}$, the mjan genome has the correspondent basic string length $p=d_{b}$ for periodic nucleotide strings. For example, the nucleotide string $\sum_{l}=a t^{2} \ldots a^{2} t(237122-237620)$ with $L_{\sum l}=499$ is formed by repeating the basic string $a t^{2} \ldots t^{2} c$ with the length $p=d_{b}$, where $L_{\sum 1}=7 p+30$. In other words, the basic string is duplicated to the positions with the correlation distances $p$, $2 p, 3 p, 4 p, 5 p, 6 p$ and $7 p$. Despite possible contribution from such periodic nucleotide strings, the periodic correlation structure is mainly formed by the transference of nonperiodic nucleotide strings, which has approximately the same increasing period. For example, the nucleotide string $\sum_{2}=a^{2} t^{2} a^{4}$ tcagac $\mathrm{gt}^{3} \mathrm{cg}^{2} a^{2} \operatorname{tg}^{2} a^{3}(447-476)$ with $L_{\sum 2}=30$ is transferred to the places (514-543), (581-610), (651-680), (718-747), (785-814), (855-884), (922-951), (994-1023), (1064-1093), (1132-1161) and ... with the correlation distances $d_{b}, 2 d_{b}, 3 d_{b}+3,4 d_{b}+3,5 d_{b}+3,6 d_{b}+6, \quad 7 d_{b}+6$, $8 d_{b}+11,9 d_{b}+14,10 d_{b}+15$ and $\ldots$, respectively. Since the nucleotide string $\sum_{2}$ is neither periodic nor a part of a periodic nucleotide string, its periodic transference is not a repetition of basic periodic nucleotide strings. Moreover, Fig. (2) shows that there also exists a cluster of basic periodic lengths close to $d_{b}$. Their integer multiples are distributed near the periodic correlation structure. Table 2 shows that there also exists another basic string length $p=68$ for periodic nucleotide strings, which is conducible to form the cluster distribution near the periodic correlation structure. So both the repetition of basic periodic nucleotide strings and the transference of non-periodic nucleotide strings would form the periodic correlation structure with approximately the same increasing period.

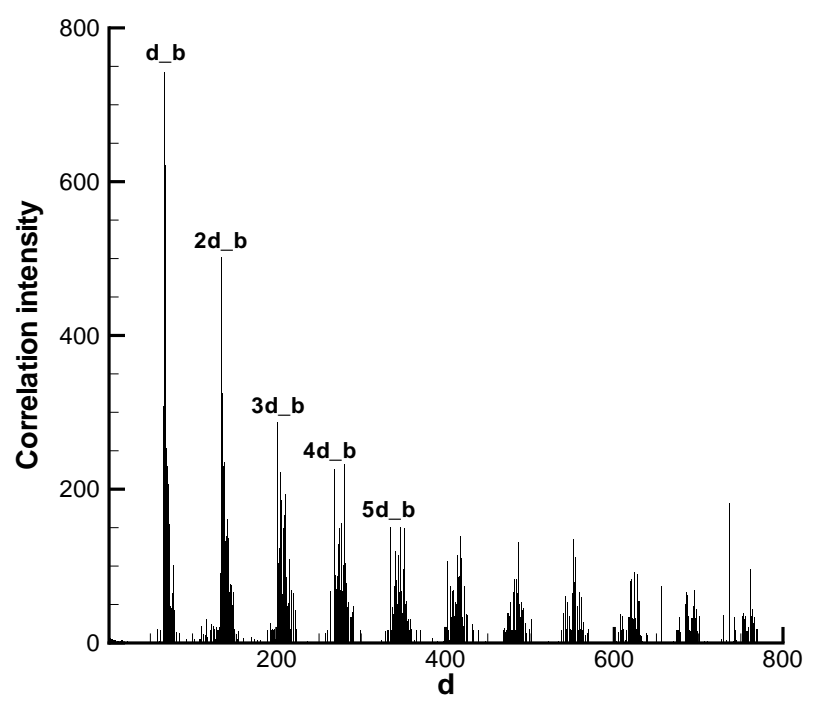

Fig. (2). A plot of correlation intensity $\Xi(d)$ versus correlation distance $d$ for the Methanococcus jannaschii DSM 2661 (mjan) genome.

Besides the mjan genome, the other genomes (mthe, pyro, aful, pabyssi and tmar) have no periodic nucleotide strings with the basic string length $p=d_{b}$ to make contributions to the periodic correlation structure. So the periodic correlation structure is formed by the transference of non-periodic nucleotide strings. Furthermore, the genomes of Mycoplasma genitalium G37 (mgen), hinf, Mycoplasma pneumoniae M129 (mpneu), Treponema pallidum subsp. pallidum str. nichols (tpal), Aeropyrum pernix K1 (aero), Rickettsia prowazekii str. madrid E (rpxx) and Borrelia burgdorferi B31 (bbur) have basic periodic lengths $d_{b}=3,4$, $12,24,65,84$ and 162, respectively. In Table 2 , they correspond to periodic nucleotide strings with the basic length $p=d_{b}$ except the aero genome. So both the repetition of basic periodic nucleotide strings and the transference of non-periodic nucleotide strings would form the periodic correlation structure with approximately the same increasing period.

(2) The correlation distance contains several increasing periods. The Escherichia coli K-12 MG1655 (ecoli) genome has two basic periodic lengths 100 and 113. The hpyl99 genome has three basic periodic lengths 8,15 and 21 . The $m t u b$ genome has three basic periodic lengths 9,15 and 57. Consider the hpyl99 genome as an example. Fig. (3) displays the correlation intensity at different correlation distances with $l=15$ for the hpyl99 genome. It is evident that there exist some equidistant parallel lines with basic periodic lengths, to form periodic correlation structures. Three basic periodic lengths are determined as $d_{b 1}=8, d_{b 2}=15$ and $d_{b 3}=21$. Although there are some peaks of the correlation intensity in the correlation distance as shown in Fig. (3), they 
do not form any periodic correlation structures and are not accounted. Table 2 also shows some periodic nucleotide strings with basic string lengths $p_{1}=d_{b 1}, p_{2}=d_{b 2}, p_{3}=d_{b 3}$ and their integer multiples, which contribute to the periodic correlation structures. For example, the nucleotide string $\sum_{I}=c a^{2} \ldots c a^{2}(1061079-1061153)$ with $L_{\sum l}=75$ is formed by repeating the basic string $c a^{2} c^{2} a t^{2}$ with the length $p_{1}$, where $L_{\Sigma l}=9 p_{1}+3$. The nucleotide string $\sum_{2}=a^{3} \ldots c a^{2}(5153-5280)$ with $L_{\sum_{2}}=128$ is formed by repeating the basic string $\mathrm{a}^{5} \mathrm{ca}^{3} \mathrm{ga}^{2} \mathrm{t}^{3}$ with the length $p_{2}$, where $L_{\Sigma 2}=8 p_{2}+8$. The nucleotide string $\sum_{3}=a t c \ldots t c a \quad(659300-659450)$ with $L_{\sum 3}=151$ is formed by repeating the basic string atcata $t^{2} a^{2} c^{3} t c a^{3} t c$ with the length $p_{3}$, where $L_{\sum_{3}}=7 p_{3}+4$. Although the transference of non-periodic nucleotide strings might also contribute to the periodic correlation structures, they are mainly formed by repeating the basic periodic nucleotide strings. For example, the non-periodic nucleotide string $\sum_{4}=a g a^{4} c^{2} t^{2} c t a^{3} g a^{6} c$ (59514-59537) with $L_{\Sigma 4}=24$ is transferred to the places (59640-59663) and (59724-59747) with the correlation distances $6 d_{b 3}$ and $10 d_{b 3}$, respectively. So both the repetition of basic periodic nucleotide strings and the transference of non-periodic nucleotide strings would form the periodic correlation structures with approximately the same increasing periods.

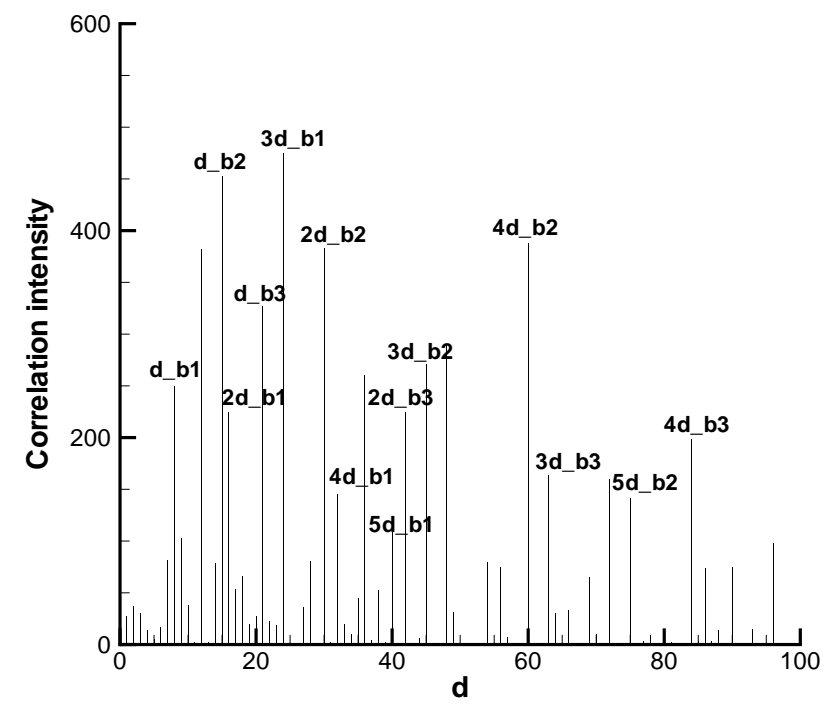

Fig. (3). A plot of correlation intensity $\Xi(d)$ versus correlation distance $d$ for the Helicobacter pylori J99 (hpyl99) genome.

(3) The correlation distance has an increasing quasiperiod. The Bacillus subtilis subsp. subtilis str. 168 (bsub) genome has a basic quasi-periodic length of 5000. Fig. (4) shows the correlation intensity at different correlation distances with $l=15$ for the $b s u b$ genome. It is evident that there exist some approximately equidistant parallel lines at the positions $d=4996,10605,15427$ and 20468 , to form a quasi-periodic correlation structure with a basic quasiperiodic length $d_{b} \approx 5000$. Although a stronger correlation intensity appears at the position $d=5856$, it is far away from the quasi-periodic correlation structure and is not accounted. In Table 2, there are no periodic nucleotide strings with the length $p=d_{b}$ to make a contribution to the quasi-periodic correlation structure. For example, the non-periodic nucleotide string $\sum_{l}=a g c \ldots t a c \quad(167978-169382) \quad$ with $L_{\Sigma I}=1405$ is transferred to the place (172974-174378) with the correlation distance 4996. The non-periodic nucleotide string $\sum_{2}=t^{3} \ldots c t^{2} \quad(161449-161666)$ with $L_{\sum_{2}}=218$ is transferred to the places (167057-167274), (172056-172273) and (946761-946798) with the correlation distances 5608, 10607 and 785321, respectively. So the transference of nonperiodic nucleotide strings would form the quasi-periodic correlation structure.

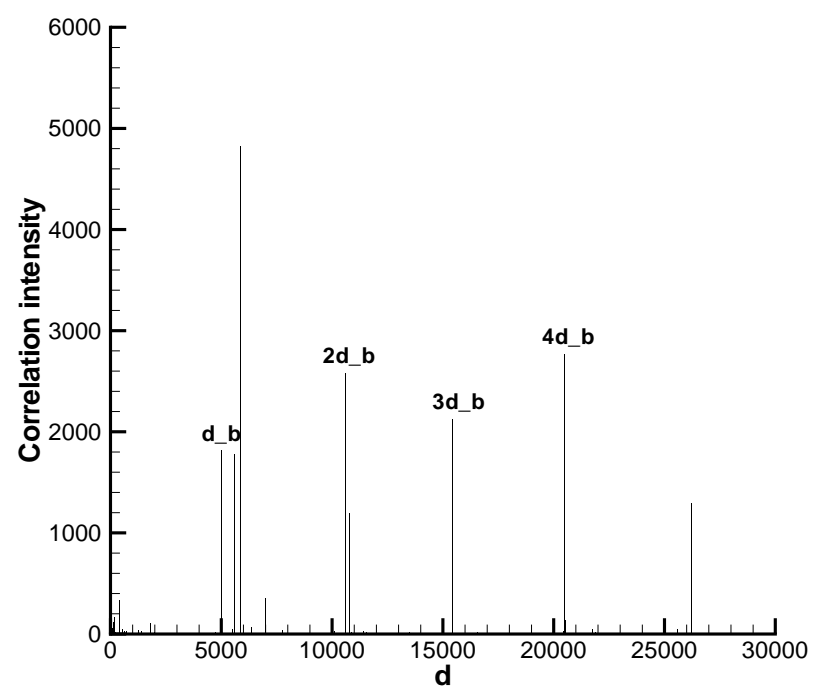

Fig. (4). A plot of correlation intensity $\Xi(d)$ versus correlation distance $d$ for the Bacillus subtilis subsp. subtilis str. 168 (bsub) genome.

(4) The correlation distance contains a combination of several increasing periods and an increasing quasi-period. Firstly, the $h p y l$ genome has two basic periodic lengths 7, 8 and a basic quasi-periodic length of 114. Fig. (5a) shows the correlation intensity at different correlation distances with $l=15$ for the $h p y l$ genome, with a local region magnified. It is evident that there exist some equidistant parallel lines with basic periodic lengths, to form periodic correlation structures in a short range of the correlation distance. The two basic periodic lengths are determined as $d_{b 1}=7$ and $d_{b 2}=8$. Moreover, in Fig. (5a), there also exist some approximately equidistant parallel lines at the positions $d=96,207,324$, $438,552,666$ and 780 , to form a quasi-periodic correlation structure in a long range of the correlation distance. The quasi-periodic correlation distance is described as $d \approx 96+x d_{b 3}$, where the basic quasi-periodic length $d_{b 3}$ is 114 and $x=0,1,2, \ldots$ In Table 2, there exist some periodic nucleotide strings with basic string lengths $p_{1}=d_{b 1}, p_{2}=d_{b 2}$ and their integer multiples, but no periodic nucleotide strings with the basic string length $p_{3}=d_{b 3}$. For example, the nucleotide string $\sum_{1}=\operatorname{tg} a \ldots t^{2} a(1-181)$ with $L_{\Sigma l}=181$ is formed by repeating the basic string $\operatorname{tgat}^{2} a g$ with the length $\mathrm{p}_{1}$, where $L_{\Sigma l}=25 p_{1}+6$. The nucleotide string $\sum_{2}=t^{2} g \ldots \operatorname{tg} a$ (444403-444490) with $L_{\Sigma 2}=88$ is formed by repeating the basic string $t^{2} g c t^{2} g a$ with the length $\mathrm{p}_{2}$, where $L_{\Sigma 2}=11 p_{2}$. Although the transference of non-periodic nucleotide strings might also contribute to the periodic correlation structures, they are mainly formed by repeating the basic periodic nucleotide strings. For example, the non-periodic nucleotide string $\sum_{3}=$ atg tatg $^{2}$ catg $^{3}$ catgtatg $(84905-84926)$ with $L_{\Sigma 3}=22$ is transferred to the place (84929-94850) with the correlation distance $3 d_{b 2}$. Moreover, for the quasi-periodic correlation structure, the non-periodic nucleotide string $\sum_{4}=$ gtgagta $^{2}$ $t^{3}{ }^{3} t c g c a t^{2} c t^{6} c t c$ (556196-556224) with $L_{\sum 4}=29$ is transferred 
to the places $(556634-556662),(556748-556776),(556862-$ $556906)$, (557300-557328), (557414-557442) and (557852$557880)$ with the correlation distances $96+3 d_{b 3}, 96+4 d_{b 3}$, $96+5 d_{b 3}, 78+9 d_{b 3}, 78+10 d_{b 3}$ and $60+14 d_{b 3}$, respectively. So both the repetition of basic periodic nucleotide strings and the transference of non-periodic nucleotide strings would form the periodic correlation structures with approximately the same increasing periods in a short correlation distance, but only the transference of non-periodic nucleotide strings would form the quasi-periodic correlation structure in a long correlation distance.

Secondly, the synecho genome has two basic periodic lengths 6, 888 and a basic quasi-periodic length of 296. Fig. (5b) shows the correlation intensity at different correlation distance with $l=15$ for the synecho genome, with a local region magnified. It is evident that there exist some equidistant parallel lines with basic periodic lengths, to form periodic correlation structures in short and long ranges of correlation distances, respectively. Two basic periodic lengths are determined as $d_{b 1}=6$ and $d_{b 2}=888$. Moreover, in Fig. (5b), there also exist some approximately equidistant parallel lines at the positions $d_{1}=297+x_{1} d_{b 2} \approx\left(1+3 x_{1}\right) d_{b 3}$ and $d_{2}=591+x_{2} d_{b 2} \approx\left(2+3 x_{2}\right) d_{b 3}$, where the basic quasi-periodic length $d_{b 3}$ is 296 and $x_{1}, x_{2}=0,1,2, \ldots$ They form quasiperiodic correlation structures in a long range of the correlation distance. In Table $\mathbf{2}$, there exist some periodic nucleotide strings with integer multiples of $d_{b 1}$ and the basic string length $p_{2}=d_{b 2}$, but no periodic nucleotide strings with the basic string length $p_{3}=d_{b 3}$. For example, the nucleotide string $\sum_{1}=$ gag ...tga (527703-527770) with $L_{\sum 1}=68$ is formed by repeating the basic string $g a g c^{2} g^{2} a^{2} c^{2} \operatorname{tg} a^{2} c^{3}$ with the length $p_{1}=3 d_{b l}$, where $L_{\Sigma l}=3 p_{1}+14$. The nucleotide string $\sum_{2}=c a c \ldots g^{2} t(2354010-2355833)$ with $L_{\sum 2}=1824$ is formed by repeating the basic string $c a c \ldots c a t$ with the length $p_{2}$, where $L_{\Sigma_{2}}=2 p_{2}+48$. Moreover, the nucleotide string $\sum_{3}=\left(\operatorname{ctg} a^{2} c^{3} g a g c^{2} g^{2} a^{2} c\right)^{2} \operatorname{ctg} a(527395-527434)$ with $L_{\sum 3}=40$ is transferred to the places $(527473-527512)$, (527491$527530)$, (527509-527548), (527527-527566) and (527545$527584)$ with the correlation distances $13 d_{b 1}, 16 d_{b 1}, 19 d_{b 1}$, $22 d_{b 1}$, and $25 d_{b 1}$, respectively. The non-periodic nucleotide string $\sum_{4}=c a c \ldots t c g(2354010-2354300)$ with $L_{\sum 4}=291$ is transferred to the places (2356674-2356964), (23575622357852) and (2358450-2358740) with the correlation distances $3 d_{b 2}, 4 d_{b 2}$ and $5 d_{b 2}$, respectively. Both the repetition of basic periodic nucleotide strings and the transference of non-periodic nucleotide strings would form the periodic correlation structures with approximately the same increasing periods in short and long correlation distances, but only the transference of non-periodic nucleotide strings would form the quasi-periodic correlation structures in a long correlation distance.

(5) The correlation distance contains almost no increasing periods. The genomes of Aquifex aeolicus VF5 (aquae), Rhizobium sp. NGR234 plasmid pNGR234a (pNGR234), Chlamydophila pneumoniae CWL029 (cpneu) and Chlamydia trachomatis D/UW-3/CX (ctra) are among cases with such characteristics. Consider the aquae genome as an example. Fig. (6) shows the correlation intensity at different correlation distances with $l=15$ for the aquae genome. It is evident that there exist some equidistant parallel lines with a basic periodic length, which is determined as $d_{b}=67$. However, for the basic periodic length $d_{b}=67$, the maximal correlation intensity $\Xi(d)$ is only 179 and the correlation structure has only three peaks of the correlation intensity at the positions $d_{b}, 2 d_{b}$ and $3 d_{b}$. The weak correlation intensity with a few peaks in the correlation distance may not make any periodic correlation structures. In Table 2, there are also no periodic nucleotide strings for the almost non-periodic correlation structure. So the aquae genome almost has no periodic correlation structures.
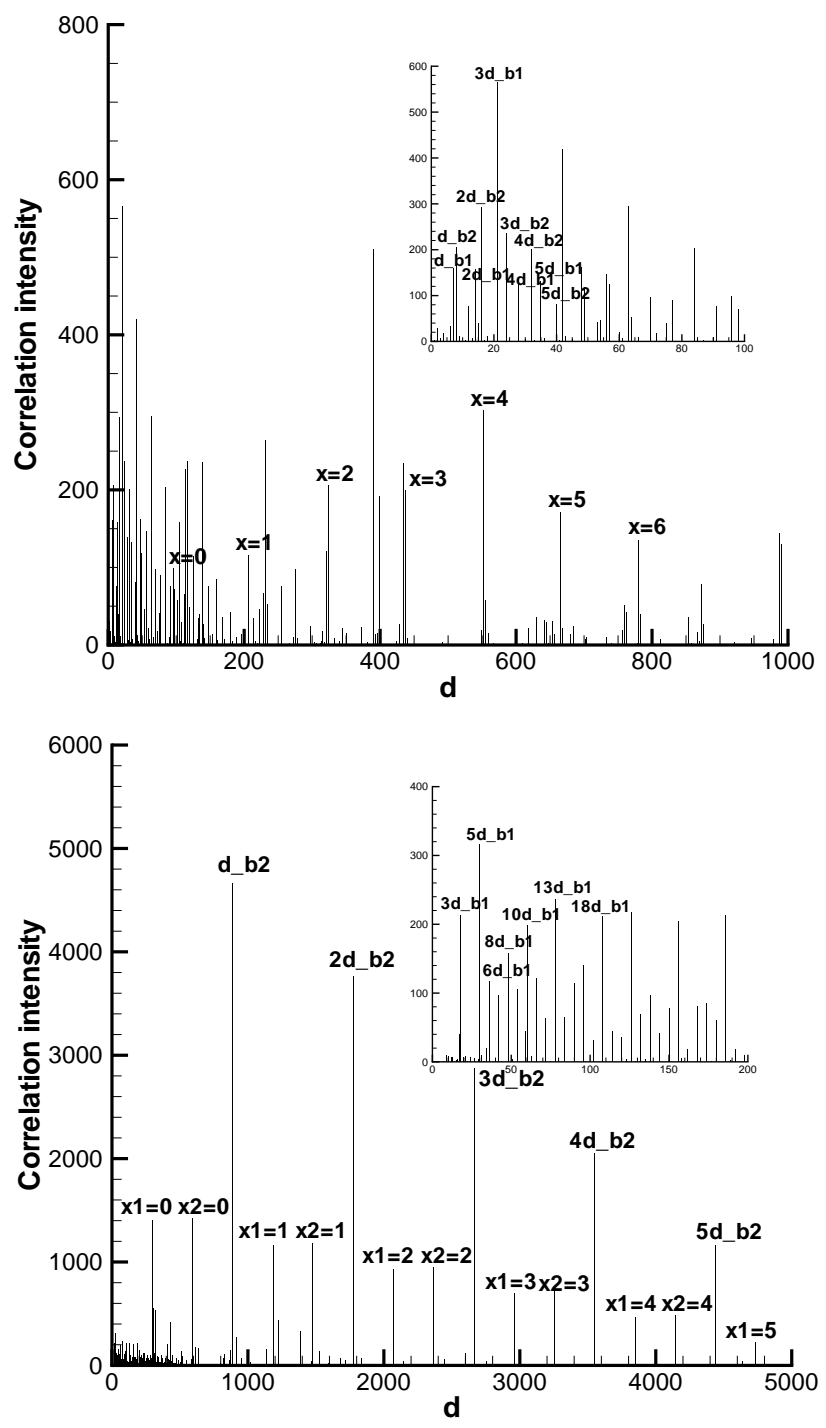

Fig. (5). Plots of correlation intensity $\Xi(d)$ versus correlation distance $d$ for (a) the Helicobacter pylori 26695 (hpyl) and (b) the Synechocystis sp. PCC6803 (synecho) genomes.

\section{CONCLUSION AND DISCUSSIONS}

In summary, using the metric representation and the recurrence plot method, we have observed periodic correlation structures in bacterial and archaeal complete genomes. All basic periodic lengths in the periodic correlation structures are determined. On the basis of the periodic correlation structures, the bacterial and archaeal complete genomes, as classified into five groups, display four kinds of fundamental transferring characteristics: a 
single increasing period, several increasing periods, an increasing quasi-period and almost noincreasing period. The mechanism of the periodic correlation structures is further analyzed by determining all long periodic nucleotide strings in the bacterial and archaeal complete genomes and is explained as follows: both the repetition of basic periodic nucleotide strings and the transference of non-periodic nucleotide strings would form the periodic correlation structures with approximately the same increasing periods.

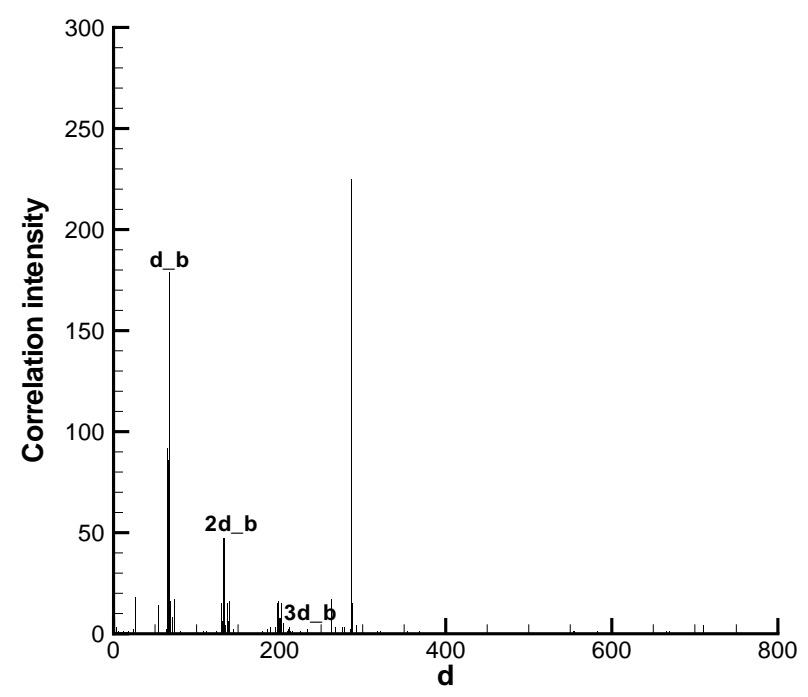

Fig. (6). A plot of correlation intensity $\Xi(d)$ versus correlation distance $d$ for the Aquifex aeolicus VF5 (aquae) genome.

In comparison with the complete genome of the Saccharomyces cevevisiae yeast [32], it is found that the bacterial, archaeal and yeast complete genomes have the same four kinds of fundamental transferring characteristics of nucleotide strings. They choose preferably the basic periodic length $d_{b} \approx 67$ or its double $d_{b} \approx 135$ in the periodic correlation structures, even they do not have basic string lengths of long periodic nucleotide strings, which are equal to the basic periodic lengths. The basic periodic length $d_{b} \approx 135$ was also found in the correlation analysis of the human genomes [10].

Although more and more biological functions of the junk DNA in cells are found, the mystery of transposable elements in the whole genomes remains unraveled. The purpose of this work is to depict the genome structure in the bacterial and archaeal complete genomes and explain the genome dynamics in terms of nucleotide string transfer. The proposed periodic correlation structures with approximately the same increasing periods may have fundamental importance for the biological functions of the junk DNA.

\section{CONFLICT OF INTEREST}

The authors confirm that this article content has no conflict of interest.

\section{ACKNOWLEDGEMENTS}

We would like to thank the National Science Foundation for partial support through the Grant No. 11172310 and the
IMECH/SCCAS SHENTENG 1800/7000 research computing facility for assisting in the computation.

\section{REFERENCES}

[1] Wille LT. New Directions in Statistical Physics: Econophysics, Bioinformatics, and Pattern Recognition. Springer Press: 2004.

[2] Peyrard M. Nonlinear dynamics and statistical physics of DNA. Nonlinearity 2004; 17: R1-R40.

[3] Jeffrey HJ. Chaos game representation of gene structure. Nucleic Acids Res 1990; 18: 2163-70.

[4] Li W, Kaneko K. Long-range correlation and partial 1/f spectrum in a non-coding DNA sequence. Europhys Lett 1992; 17: 655-60.

[5] Peng CK, Buldyrev SV, Goldberger AL, et al. Long-range correlations in nucleotide sequence. Nature 1992; 356: 168-70.

[6] Deschavanne PJ, Giron A, Vilain J, Fagot G, Fertil B. Genomic signature: characterization and classification of species assessed by chaos game representation of sequences. Mol Biol Evol 1999; 16: 1391-9.

[7] Hao BL. Fractals from genomes - exact solutions of a biologyinspired problem. Physica A 2000; 282: 225-46.

[8] Qi D, Cuticchia AJ. Compositional symmetries in complete genomes. Bioinformatics 2001; 17: 557-9.

[9] Bernaola-Galván P, Carpena P, Román-Roldán R, Oliver JL. Study of statistical correlations in DNA sequences. Gene 2002; 300: 10515 .

[10] Holste D, Grosse I, Beirer S, Schieg P, Herzel H. Repeats and correlations in human DNA sequences. Phys Rev E 2003; 67: 061913.

[11] Garte S. Fractal properties of the human genome. J Theor Biol 2004; 230: 251-60.

[12] Vinogradov AE. Noncoding DNA, isochores and gene expression: nucleosome formation potential. Nuc Asids Res 2005; 33: 559-63.

[13] Katsaloulis $\mathrm{P}$, Theoharis $\mathrm{T}$, Zheng WM, et al. Long-range correlations of RNA polymerase II promoter sequences across organisms. Physica A 2006; 366: 308-22.

[14] Vaillant C, Audit B, Arneodo A. Experiments confirm the influence of genome long-range correlations on nucleosome positioning. Phys Rev Lett 2007; 99: 218103.

[15] Sindi SS, Hunt BR, Yorke JA. Duplication count distributions in DNA sequences. Phys Rev E 2008; 78: 061912.

[16] Kong SG, Fan WL, Chen HD, Wigger J, Torda AE, Lee HC. Quantitative measure of randomness and order for complete genomes. Phys Rev E 2009; 79: 061911.

[17] Provata A, Katsaloulis P. Hierarchical multifractal representation of symbolic sequences and application to human chromosomes. Phys Rev E 2010; 81: 026102.

[18] Bershadskii A. Coherence and stacking interaction in DNA duplex. Phys Lett A 2011; 375: 3021-4.

[19] Bennetzen JL. Transposable element contributions to plant gene and genome evolution. Plant Mol Biol 2000; 42: 251-69.

[20] Feschotte C, Jiang N, Wessler SR. Plant transposable elements: where genetics meets genomics. Nat Rev Genet 2002; 3: 329-41.

[21] Kazazian HH. Mobile elements: drivers of genome evolution. Science 2004;303 : 1626-1632.

[22] Bergman CM, Quesneville H. Discovering and detecting transposable elements in genome sequences. Brief Bioinform 2007; 8: 382-92.

[23] Lönnig WE, Saedler H. Chromosome rearrangements and transposable elements. Ann Rev Genet 2002; 36: 389-410.

[24] Shapiro JA. Mobile DNA and evolution in the $21^{\text {st }}$ century. Mobile DNA 2010; 1: 1-14.

[25] Hao BL, Zheng WM. Applied Symbolic Dynamics and Chaos. World Scientific, Singapore: 1998.

[26] Eckmann JP, Kamphorst SO, Ruelle D. Recurrence Plots of Dynamical Systems. Europhys Lett 1987; 5: 973-77; Marwan N, Romano MC, Thiel M, Kurths J. Recurrence plots for the analysis of complex systems. Phys Rep 2007; 438: 237-329.

[27] Guastello SJ. Progress of Applied Nonlinear Dynamics: Welcome to NDPLS volume 8. Nonlinear Dynam Psychol Life Sci 2004; 8: $1-15$.

[28] Spinelli G. Heterochromatin and complexity: A theoretical approach. Nonlinear Dynam Psychol Life Sci 2003; 7: 329-61.

[29] Wu ZB. Metric representation of DNA sequences. Electrophoresis 2000; $21: 2321-6$. 
[30] Wu ZB. Self-similarity limits of genomic signatures. Fractals 2003; 11: 19-25.

[31] Wu ZB. Recurrence plot analysis of DNA sequences. Phys Lett A 2004; $232: 250-5$.
[32] Wu ZB. Global transposable characteristics in the complete DNA sequence of the yeast. Physica A 2010; 389: 5698-705. 